\title{
The Dust Management Project: Characterizing Lunar Environments and Dust, Developing Regolith Mitigation Technology and Simulants
}

\author{
Mark J. Hyatt ${ }^{1}$ \\ NASA John H. Glenn Research Center at Lewis Field, Cleveland, OH, 44135, USA \\ and \\ Sharon A. Straka ${ }^{2}$ \\ NASA Goddard Space Flight Center, Greenbelt, MD, 20771, USA
}

\begin{abstract}
A return to the Moon to extend human presence, pursue scientific activities, use the Moon to prepare for future human missions to Mars, and expand Earth's economic sphere, will require investment in developing new technologies and capabilities to achieve affordable and sustainable human exploration. From the operational experience gained and lessons learned during the Apollo missions, conducting long-term operations in the lunar environment will be a particular challenge, given the difficulties presented by the unique physical properties and other characteristics of lunar regolith, including dust. The Apollo missions and other lunar explorations have identified significant lunar dust-related problems that will challenge future mission success. Comprised of regolith particles ranging in size from tens of nanometers to microns, lunar dust is a manifestation of the complex interaction of the lunar soil with multiple mechanical, electrical, and gravitational effects. The environmental and anthropogenic factors effecting the perturbation, transport, and deposition of lunar dust must be studied in order to mitigate it's potentially harmful effects on exploration systems and human explorers. The Dust Management Project (DMP) is tasked with the evaluation of lunar dust effects, assessment of the resulting risks, and development of mitigation and management strategies and technologies related to Exploration Systems architectures. To this end, the DMP supports the overall goal of the Exploration Technology Development Program (ETDP) of addressing the relevant high priority technology needs of multiple elements within the Constellation Program (CxP) and sister ETDP projects. Project scope, plans, and accomplishments will be presented.
\end{abstract}

\section{Introduction}

A return to the Moon to extend human presence, pursue scientific activities, use the Moon to prepare for future human missions to Mars, and expand Earth's economic sphere, will require investment in developing new technologies and capabilities to achieve affordable and sustainable human exploration. From the operational experience gained and lessons learned during the Apollo missions, conducting long-term operations in the lunar environment will be a particular challenge, given the difficulties presented by the unique physical properties and other characteristics of lunar regolith, including dust. The Apollo missions and other lunar explorations have identified significant lunar dust-related problems that will threaten future mission success. ${ }^{1,2}$ The DMP is tasked with the evaluation of lunar dust effects, assessment of the resulting risks, and development of mitigation and management strategies and technologies related to Exploration Systems architecture.

Specific project objectives for the DMP include:

- Address high priority technology needs, customer and derived requirements.

\footnotetext{
${ }^{1}$ Engineering Project Manager, Advanced Capabilities Projects Office, 21000 Brookpark Rd., AIAA Member.

${ }^{2}$ Lead Aerospace Engineer, Spacecraft Coatings and Contamination Group, Mailstop 546.0.
} 
- Provide knowledge and technologies (to Technology Readiness Level-6 (TRL-6) development level) required to address adverse dust effects to humans and to exploration systems and equipment, which will reduce life cycle cost and risk, and will increase the probability of sustainable and successful lunar missions.

- Provide coordination across ESMD of lunar dust related activities and information to maximize benefit from Agency investments.

- Develop a balance of near- and long-term knowledge and technology development, driven by ESMD needs and schedule requirements, aligned with available technology investments where possible.

- Leverage existing Agency capabilities, resources, and invest prudently to support the development of capabilities, as appropriate, where there are gaps.

- Based on the user's needs, develop appropriate, affordable, and timely soil simulants for NASA's Exploration Program to use in risk reduction.

To this end, the Dust Management Project (DMP) supports the overall goal of the Exploration Technology Development Program (ETDP) of addressing the relevant high priority technology needs of multiple elements within the Constellation Program (CxP) and sister ETDP projects. The DMP will provide needed knowledge and understanding of the lunar environment with respect to interactions between lunar regolith and surface system hardware. DMP identifies and evaluates capabilities and new technologies that can provide significant mass, cost, operability, and/or risk reduction attributes. The DMP develops and demonstrates technologies and systems with other surface system elements, and ensures that the technologies and systems are inserted into flight system development activities at the appropriate time with the minimum risk to flight development and operation on the lunar surface. The DMP provides coordination of lunar dust related activities and information to maximize benefit from Agency investments. Additionally, the DMP develop appropriate lunar regolith simulants for the testing of surface system hardware.

\section{Technical Approach}

The DMP has developed a comprehensive research, technology, and systems development approach to meet the specific needs and requirements identified for Exploration Systems for Lunar Surface Systems (LLS), Lunar Lander Project Office (LLPO), Orion Crew Vehicle, and Extravehicular Activity (EVA). It also includes required characterization of lunar regolith to inform the engineering design process for these systems, the definition of requirements and characterization of existing and future lunar regolith simulants, and the development and production of Highland and Mare simulant types. Each element has been selected for its potential benefits in lunar dust environment knowledge generation, dust management/mitigation, or environmental testing. The DMP technology portfolio is organized with respect to multi-application technology development areas (Electrodynamic Dust Shield, Lotus Coating, SPARCLED, and $\mathrm{CO}_{2}$ Shower), specific technology focus areas (Mechanical Components, Thermal Control Surfaces, Connectors, and Surface Power), and Simulants. Detailed descriptions for each of the technology elements, technology application focus areas, and simulant development are summarized below.

\section{A. Technology Development Areas}

\section{Electrodynamic Dust Shield (EDS)}

This task is focused on the development of EDS technology to minimize dust accumulation on surfaces exposed to the external lunar environment and affected by lunar surface operations. The non-mechanical, electrodynamic cleaning method removes dust particles and prevents dust accumulation on surfaces by applying a multi-phase traveling electric field to electrodes that are embedded in the surface to lift and transport charged and uncharged particles off and away from the surface. ${ }^{1}$ The technology is currently at a TRL-3.

The EDS technology has applicability to surfaces exposed to the external lunar environment that need protection, including surface power systems (solar panels, energy storage battery modules, interface cold plates), optical systems (windows, view ports, cameras, spectrometers, light sources), thermal radiators, connectors (quick disconnects and umbilical systems), ISRU sample recovery chambers and steel plates for ISRU production plant design, and sealing surfaces of the airlock hatch and suit port. 

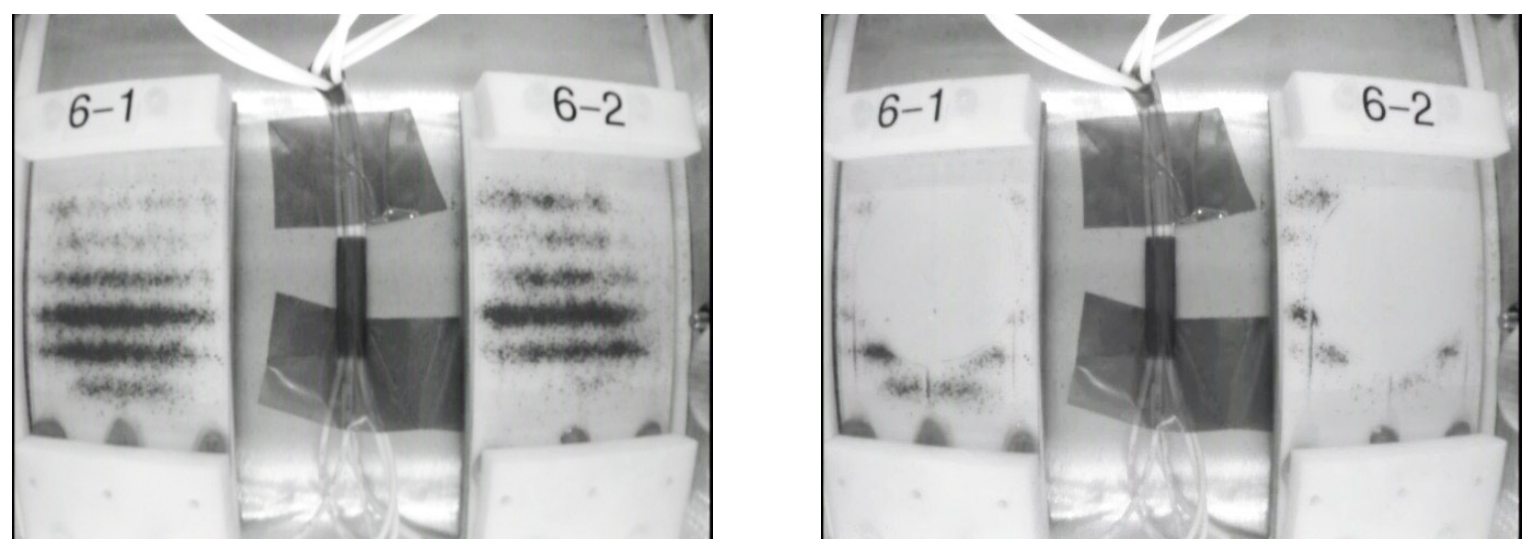

Figure 1. Dust removal demonstration by EDS with Apollo 16 lunar samples at $1 / 6 \mathrm{~g}$ and at $10^{-6} \mathrm{kPa}$

The selection of specific lunar surface system applications for development of the EDS technology has been based on several factors: customer prioritization of technology needs, current maturation of the EDS technology, analog testing opportunities and customer driven requests, level of development effort required for a specific application to achieve TRL-6 deliverables by critical customer milestone dates, and available funding (ETDP, IPP, CDDF, other sources).

The EDS component technologies can be tailored for the intended application by modifying and optimizing the design, materials, processes, and coatings to maximize shield performance, and also varying electrode spacing, electrode width, number of phases, signal frequency, signal amplitude, dust material, and dust size fraction. The EDS component technologies include the following:

- Electrode design — shape, geometry, width, spacing, amplitude, frequency, number of phases

- Shield materials—electrode (opaque/transparent, rigid/flexible) for given substrate (opaque/transparent)

- Shield processes-deposition parameters and electrode pattern formation (sputter coating or chemical etching/laser etching)

- Dust repellant insulating coatings - to protect the electrodes from the harsh lunar environment and to prevent electrical breakdown between electrodes upon application of high voltage waveforms

Candidate materials must possess the desired combination of physical, mechanical, and electrical properties, lunar environmental durability, and must be amenable to processing into the desired form (i.e., film coatings adhesive, composite, molded shapes, gaskets, etc) for integration into specific lunar dust mitigation and/or cleaning related applications. Figure 1 shows a flight demonstration of dust removal from an optically transparent surface by EDS.

\section{Lotus Coating}

The Lotus Coating task will focus primarily on the formulation, characterization and space environmental testing of the "Lotus" dust mitigation coating for space flight application. Secondary objectives of this task are to develop dust removal/cleaning methods that do not require the use of water and obtain a more comprehensive understanding of the Lunar dust environment (electrostatic properties) to tailor the coating's properties or capabilities for enhanced survivability in that environment. This technology is being developed as a countermeasure for dust accumulation during long-duration human space exploration. Figure 2 shows the effectiveness of Lotus coating, applied to a typical radiator surface, at preventing contamination by lunar dust simulant.

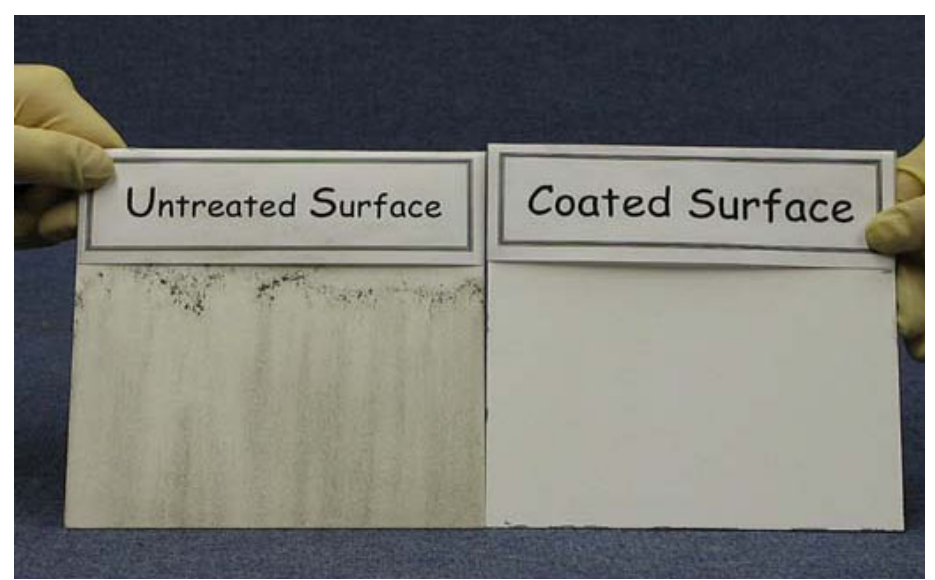

Figure 2. Uncoated radiator sample and a Lotus coated radiator sample after being contaminated with JSC-1 Lunar simulant 
Prior research and development activities have proven the concept of the Lotus coating, as a dust mitigation tool, is viable and achievable. The technology is currently at a TRL-3. The Lotus coating is microscopically thin and optically transparent. This coating sheds dust particles utilizing anti-contamination and self-cleaning properties that minimize dust accumulation on spacecraft surfaces. Upon completion of the Lotus coating formulation process, the coating will consists of the following characteristics to mitigate dust accumulation: anti-contamination properties, self-cleaning properties, environmental stability or controlled material property degradation, good adhesion, durability and clean-ability. Lotus coating formulation development will consist of utilizing a "wet chemistry" nanostructure process for in-house application. Upon the establishment of the wet chemistry application process, formulation optimization and characterization testing will commenced. Lotus coating development will also incorporate a parallel coating application effort using chemical combustion vapor deposition (CCVD) process. Viable formulations will then be applied to subsystem level components and tested in an environmental representative of the intended lunar environment to demonstrate this technology's maturity from its current TRL-3 to a TRL-6.

The Lotus Coating technology has cross cutting applications. The Lotus coating is designed to preserve optimal long-term performance of spacecraft and habitation components and systems. External lunar environment applications include: critical spacecraft thermal control surfaces [such as thermal radiators and outer layer of MultiLayer Insulation (MLI) blankets], solar array panels, optics, windows, lenses, mechanism shields, EVA materials, EVA tools, astronaut visors, and rover components. Internal lunar habitation applications could consist of: airlock, interior crew module walls, and optics/mirrors. The Lotus coating presently can be applied to metal, glass and graphite epoxy composite substrates. Future research will expand application to various substrates. For added capabilities, this technology also has the potential to be use in combination with other ETDP Dust Management technologies (such as the electrodynamic dust shield, thermal control systems, surface power systems, and ISRU optical components).

3. SPARCLED

The Space Plasma Alleviation of Regolith Concentrations in Lunar Environments by Discharge (SPARCLED) is a tool designed to remove contaminating lunar regolith from surfaces, primarily in the Lunar Habitat Airlock, but can be used in other applications. ${ }^{2}$ SPARCLED allows near radial removal of dust from surfaces, thereby minimizing abrasive tangential forces. It utilizes only electrons and hence no consumables such as gases are needed for operation. Additionally the SPARCLED electrostatic forces can be adjusted to overcome all binding forces to all surfaces: mechanical, van der Waals, capillary, existing electrostatic or magnetic. SPARCLED also provides an empirical basis for electrostatic model of grain accretion, which has application to modeling of planetary system formation processes. ${ }^{3}$

SPARCLED uses an electron gun beam to rapidly charge dust contaminating a surface to a sufficiently high charge-to-mass ratio to cause rapid and complete removal of the dust grains by mutually repulsion. The tool utilizes a Penning Discharge initiated by the application of a weak electric field in the electron beam. The resulting discharge converts the relatively high energy, low density electron flux from the gun to a low energy, high density

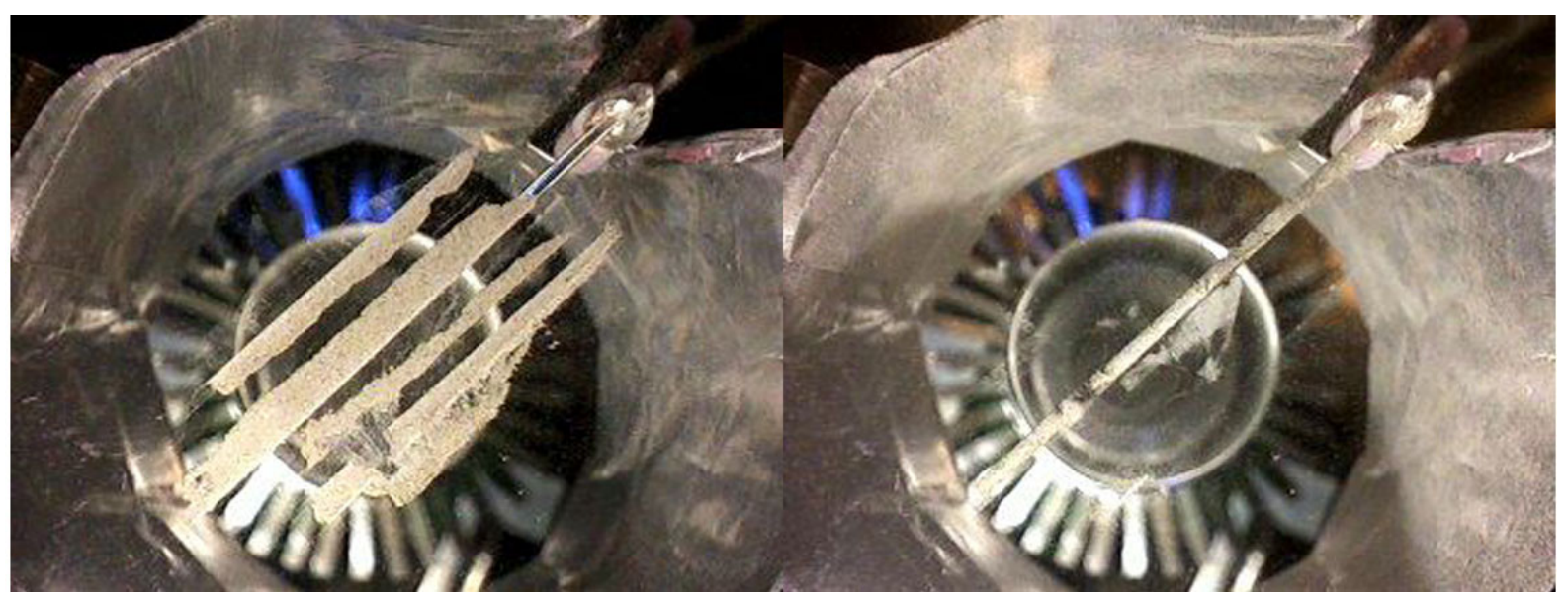

a) before (lines of JSC-1AF simulant)

b) after (dust transferred to collector)

Figure 3. Before (a) and after (b) images of SPARCLED dust extraction from a dielectric surface 
electron flux. This conversion maximizes the efficiency of dust charging and hence removal efficacy. Subsequently, SPARCLED uses charged surfaces and the discharge enhanced gun to remove dust from the contaminated surface (surface cleaning), collect it on a capture surface (dust collection), and to extract dust from collection surfaces (tool cleaning). SPARCLED easily removes with higher surface-to-volume ratio very small submicron grains with the high charge to mass ratio. Operating physics is completely scalable from lunar habitat airlock environment to Mars surface environment.

4. $\mathrm{CO}_{2}$ Shower

To protect entry to airlock and habitat, the development of deployable, possibly inflatable, dust isolation and removal zones will be conducted using $\mathrm{CO}_{2}$ snow shower technology. The approach will be to analyze the concept and identify controlling parameters of a $\mathrm{CO}_{2}$ shower. During this development phase, concentrations of $\mathrm{CO}_{2}$ and $\mathrm{N}_{2}$ will be evaluated as a function of temperature and dust removal efficiency. A study will be performed to optimize the balance between Earth provided resources and Moon recovered resources for the $\mathrm{CO}_{2}$ shower. The $\mathrm{CO}_{2}$ prototype shower will have a camera and LED lighting for observing the dust removal process. The shower system will be placed into an environmental chamber with gas, pressure and temperature control. Multiple test samples will be evaluated for each pump down sequence. By observing the dust particle movement in the shower with $0.1 \mathrm{~atm}$ of $\mathrm{CO}_{2}$ and vacuum the expected movement on the moon may be calculated. This data can be compared to actual dust movement on the moon.

\section{B. Technology Focus Areas}

\section{Mechanical Components}

The Mechanical Components task's primary function is to identify and address dust contamination issues for mechanisms, particularly seals, joints, gears, and bearings. Mechanical systems are at risk for being adversely affected by lunar dust. Component level tests are needed to determine effects of dust contamination on mechanisms, components, and lubricants. Dust tolerant bearings and drives need to be designed to survive the lunar regolith environment for long periods. Mechanism designs must also be robust enough to survive a long life in a lunar dust environment and provide nominal operation with lunar dust contamination.

A series of dust contamination investigations will be performed at the component level. Endurance tests will be conducted on mechanism and drive system components, such as gears and bearings, with various levels of dust contaminated grease. Additionally, performance tests will be performed on gear materials and coatings, seals, and mechanisms. Fundamental lubricant tests will also be run. In addition to the contamination investigations, technologies will be developed and tested to prevent dust contamination and/or mitigate its effects. These technologies include ceramic bearings, rail-race bearings, advanced bellows for joints, shaft seals, ceramic gears and coatings, and various SBIR derived technologies as they are developed and become available. These mechanical component-level technologies will then be integrated and tested to TRL-6 in a relevant environment.

2. Thermal Control Surfaces

The Thermal Control Surface task involves developing dust mitigation technologies for thermal radiators used in the external lunar environment to minimize the effects of dust accumulation on the performance of thermal control surfaces. This task is focused on lowering the risk that the dusty lunar environment will disrupt the proper functioning of thermal control surfaces for systems operating on the lunar surface. The first part of the task is to characterize the effects of dust on the surfaces, and the second part of the task is to mitigate thermal control surface performance degradation. It is the dust mitigation technology part of the task that will need to move from development to integration into thermal control systems, to infusion into lunar surface systems.

3. Connectors

The objectives of this task are component level integration and testing of connectors (quick disconnects and umbilical systems) that can be repetitively and reliably mated and de-mated during Lunar surface extra-vehicular activities. These standardized interfaces will be required for structural integrity and commodities transfer between linked surface elements. Umbilical electro-mechanical systems (connectors) are needed between discrete surface systems for transfer of air, power, fluid (water), and data and must be capable of being operated by extra vehicular astronaut crew members and/or robotic assistants. QD's fittings are needed for EVA spacesuit Primary Life Support Systems as well as liquid cooled garment circulation and suit heat rejection. EVA suit integration and requirements 
are being considered throughout the LSS infrastructure connector design process for possible near future integration to the suit itself as a secondary consideration; however, no funding is currently being provided for this interface. There exists an urgent need to prevent electro-statically charged dust and debris from clogging and degrading the interface seals and causing leakage and spills of hazardous commodities, contaminating the flow stream, and degrading the mechanisms needed for umbilical connection. Integration of electrostatic dust shields, Lotus coatings and other dust mitigation strategies are being considered to enhance the operating ability of the connectors. Other challenges include modularity, standardization, autonomous operation, and lifetime sealing issues.

\section{Surface Power}

Dust mitigation technologies for surface power system component level technologies (solar panel, energy storage battery modules, interface plates, etc.) are being developed to TRL-6 in order to address adverse lunar soil effects, which will reduce life cycle cost and risk, and increase the probability of mission success. The hardware will be integrated and tested in a relevant simulated lunar environment. Solar array samples will be tested and modified in an effort to develop design guidelines and methodology that will minimize the effects of lunar soil loading in a practical surface power system. Subscale system tests with PV array samples will optimize the system and will be used to produce engineering design guidelines. Dust repellant materials/coatings and technologies will be developed and tested on small scale component substrate surfaces. The regolith mitigation technologies will be integrated into the surface power component subsystem and prototype demonstration will be performed in a relevant environment. Applicable DMP technologies developed to address dust mitigation of surface power systems include EDS, Lotus, and SBIR developed technologies as they become available.

Additionally, an experimental task was conducted to investigate tribocharging. The objective of this task was to characterize and quantify the magnitude of triboelectric charging under lunar versus Martian surface conditions. Triboelectric charging of a moving rover wheel in JSC-1a lunar mare simulant was compared under hard vacuum and 7 torr of $\mathrm{CO}_{2}$. Charging under Mars surface conditions ( -7 torr $\mathrm{CO}_{2}$ - was 140 to $150 \mathrm{~V}$ ), is larger than the typical $100 \mathrm{~V}$ measured in the late 1990s using Arizona Lunar Simulant. The magnitude of charging under hard vacuum was more variable, averaging 200 to $400 \mathrm{~V}$, or about twice as much as with $\mathrm{CO}_{2}$ present. The resulting electrostatic charging leads to significant dust accumulation and adhesion to adjacent surfaces. Results showed that the problem will be approximately twice as severe on the Moon as on Mars. Mitigation techniques currently deployed on all Mars rovers are based on attached micro discharge point. Since these use the tenuous Martian atmosphere to bleed charge, they will be ineffective on the moon, and alternative mitigation approaches will have to be developed and evaluated.

\section{Simulants}

Because the quantity of actual lunar samples brought back from the Apollo missions is fairly small ( $842 \mathrm{lb}$ ) and is considered a national treasure, it is necessary to develop a lunar regolith substitute (or simulant) that can be used in lieu of the regolith for research and testing. Lunar simulants which replicate the appropriate properties of the lunar regolith are needed in order to perform technology investigations and developments resulting in advancements to a TRL-6. In addition, lunar simulants will also be required by hardware developers for requirements verification and hardware certification.

These efforts must be performed here on Earth prior to returning to the moon to achieve greater probability of mission success. Providing simulants for technology development and then for verification/certification of hardware is an essential two-pronged risk reduction "tool" that will be employed by multiple ETDP projects developing technologies for exploration surface systems. In order to meet this requirement, the DMP has been tasked with the development, characterization, and production of various types of lunar regolith simulants covering a range of grain sizes and properties depending upon the simulant user's test objectives and applications.

Defining and understanding simulant users test objectives, and how the simulants will be utilized, is critical in successfully developing right simulants for the right purpose(s) or applications at the right time. This requires close communication and interfacing between the Simulant Task Team and the LSS Simulant Users (as well as other CxP users). Based on the users' needs, the simulant task will develop and produce appropriate, affordable, and timely lunar regolith simulants for NASA's Exploration Program to use for risk reduction.

The simulant development effort in the DMP is currently evaluating and characterizing existing simulants, developing a "Figure of Merit" approach for quantification of the fit for purpose of simulants, and developing new simulants as appropriate, based on surveys of user needs and requirements. ${ }^{4}$ 


\section{Project Interfaces}

The DMP utilizes Project to Project Cooperative Agreements (PPCAs) with other ETDP projects to identify leveraging opportunities, consolidate trade studies as appropriate, and to support the development of integrated dust mitigation strategies across projects and systems. The DMP provides data pertinent to regolith characteristics and the lunar environment as well as develops, integrates, and operates hardware and software with the following ETDP Projects through PPCAs to ensure coordination of schedules, requirements, and hardware:

Table 1. DMP Interfaces with Other ETDP Projects Supporting LSS

\begin{tabular}{l|l}
\hline \multicolumn{1}{c|}{ ETDP Project } & \multicolumn{1}{c}{ DMP Interfaces } \\
\hline Human Robotic Systems (HRS) & $\begin{array}{l}\text { Regolith tolerant mechanical components } \\
\text { Regolith \& lunar environment data } \\
\text { Simulants for testing \& verification } \\
\text { Regolith \& lunar environment data } \\
\text { In Situ Resource Utilization (ISRU) }\end{array}$ \\
$\begin{array}{ll}\text { Simulants for testing \& verification } \\
\text { Regolith tolerant actuators \& valves } \\
\text { Thermal Control \& Management } & \begin{array}{l}\text { Simulants for testing \& verification } \\
\text { Regolith tolerant thermal control components } \\
\text { Regolith \& lunar environment data } \\
\text { Dust accumulation prediction models } \\
\text { Limulants for testing \& verification }\end{array} \\
\text { Extravehicular Activity (EVA) } & \begin{array}{l}\text { Regolith \& lunar environment data } \\
\text { Simulants for testing \& verification }\end{array} \\
\text { Suit fabric abrasion testing protocols } \\
\text { Regolith \& lunar environment data } \\
\text { Environmental Monitoring \& Control (AEMC) }\end{array}$ \\
$\begin{array}{l}\text { Simulants for testing \& verification } \\
\text { Regolith \& lunar environment data } \\
\text { Simulants for testing \& verification }\end{array}$ \\
\hline \hline
\end{tabular}

\section{Summary}

By pursuing an integrated approach of characterization, technology development, and application focus, the DMP has made significant progress in the first three years of implementation. The project provides coordination and integration of dust related activities in ESMD, with a focus on the dust related challenges associated with long duration lunar missions. The project has established a comprehensive body of data and experience and defined and filled key gaps in knowledge and capabilities. The project has developed specific technologies useful in the mitigation and control of dust and its effects on systems. New simulants are now becoming available to assess mitigation technologies and approaches as well as perform test and verification of components and systems. Critical information and technology deliverables will be provided directly to Exploration flight (Lunar Precursor Robotic and Constellation) programs. Lunar dust should be viewed as integrated environmental phenomena, the study of which must employ a coordinated, multidisciplinary approach. The Dust Management Project will certainly include elements exploiting theoretical studies, earth-bound experiments, key measurements conducted by early robotic missions, and development of "best design" rules and specific engineering solutions for systems and their components affected by lunar dust.

\section{Acknowledgments}

The authors wish to thank the members of the Dust Management Project team, for their contributions to this paper and their technical accomplishments in support of space exploration. 


\section{References}

${ }^{1}$ Gaier, J., “The Effects of Lunar Dust on EVA Systems During the Apollo Missions,” NASA/TM-2005-213610/REV1.

${ }^{2}$ Wagner, S., “The Apollo Experience Lessons Learned for Constellation Lunar Dust Management;” NASA/TP—2006213726.

${ }^{3}$ Calle, C. I., Buhler, C. R., McFall, J. L. Snyder, S. J., "Particle Removal by Electrostatic and Dieelctrophoretic Forces for Dust Control During Lunar Exploration Missions,” Journal of Electrostatics, Vol. 67, Issues 2+3, 2009, pp. 89-92.

${ }^{4}$ Clark, P. E., Curtis, S. A., Minetto, F. A., Marshall, J. R., Nuth, J. R., "SPARCLE: Electrostatic Dust Control Tool Proof of Concept,” SPESIF-10, edited by G. Patterson, AIP, Huntsville, AL, 2010.

${ }^{5}$ Curtis, S. A., Clark, P. E., Marshall, J. R., Nuth, J. A., Minetto, F. A., Calle, C. I., “Acceleration of Grains by Weak Electron Beam Driven Discharges: Implications for Solar System Formation,” Aviation Week and Space Technology, Vol. 145, No. 24, 9 Dec. 1996, pp. 44-46.

${ }^{6}$ Edmunson, J., Betts, W., Rickman, D., McLemore, C., Fikes, J., Stoeser, D., Wilson, S., Schrader, C., "NASA Lunar Regolith Simulant Program," $41^{\text {st }}$ Lunar and Planetary Science Conference, Paper \#1786, 2010. 